'A Murphy, ${ }^{2} \mathrm{VL}$ Adams, ${ }^{3} \mathrm{H}$ Hill, ' $\mathrm{C}$ Morgan, ${ }^{2} \mathrm{GJ}$ Kemp, ${ }^{4} \mathrm{LJ}$ Abernethy, ${ }^{3} \mathrm{MA}$ Turner. 'Neonatal Unit, Liverpool Women's NHS Foundation Trust; ${ }^{2}$ MARIARC; ' ${ }^{3}$ Women's and Chidren's Health, University of Liverpool; ${ }^{4}$ Clinical Radiology, Alder Hey NHS Foundation Trust, Liverpool, UK

Background and Aims To date there are no reports of intra-subject variability for intrahepatocellular lipid (IHCL) assessed using $1 \mathrm{H}$ NMR spectroscopy in neonates. This study utilized a high-field (3-Tesla) MRI scanner to perform repeated assessment of IHCL on neonates.

Methods Measurements were acquired using a $10 \times 10 \times 10 \mathrm{~mm}$ voxel during 90 seconds. IHCL was assessed using the ratio between the lipid and water peaks. The within subject coefficient of variation (WS CV) was calculated.

Results In 15 babies, MRS was measured twice in each lobe in the same region of interest. Values $>0.2$ gave reproducible results; the WS CV was $12 \%$ on the right and $3 \%$ on the left. MRS was measured once in each lobe in 32 babies (GA at birth median 36 weeks, range 24-42; GA at scan median 42 weeks, range 37-46). Among 17 babies with MRS $>0.2$ on both sides WS CV calculated between left and right for was $54 \%$ (95\% CI 50\%-58\%) and Mean (SD) was 0.73 $(0.33)$ on the right and $0.58(0.42)$ on the left $(p<0.005)$.

Conclusions Neonatal IHCL measured using $3 \mathrm{~T}$ is markedly lower than other age groups and systematically higher on the right than the left possibly reflecting the influence of lobe size and cardiac pulsation. Values $\leq 0.2$ are imprecise and should be classified as below the limit of quantification. The optimal imaging strategy is likely to use a consistent region of interest within the right lobe.

\section{SEX DIFFERENCES IN ADIPOSE TISSUE QUANTITY AND DISTRIBUTION IN NEWBORN INFANTS}

doi:10.1136/archdischild-2012-302724.0360

KM Logan, MJ Hyde, C Gale, JR Parkinson, S Jeffries, N Modi. Neonatal Medicine, Imperial College London, London, UK

Background and Aims Adipose tissue (AT) quantity and distribution influence metabolic health. In adult life women have greater total and subcutaneous AT, but men have greater internal abdominal AT (Gender Medicine, 2009; 6: 60-75). We aimed to explore AT volume and distribution in newborn male and female infants using magnetic resonance imaging (MRI).

Methods A retrospective observational study was performed, using an existing database of neonatal body composition data, to compare male and female healthy term infants.

Results

Abstract 360 Table 1 Anthropometry at scan in male and female infants

\begin{tabular}{llll}
\hline Measurement; mean (SD) & Male (n=95) & Female (n=90) & p value \\
\hline Weight $(\mathrm{g})$ & $3590(456)$ & $3475(496)$ & 0.104 \\
Length $(\mathrm{cm})$ & $53.0(2.2)$ & $52.7(2.8)$ & 0.435 \\
Head circumference $(\mathrm{cm})$ & $36.4(1.3)$ & $35.8(1.3)$ & 0.002 \\
\hline
\end{tabular}

Abstract 360 Table 2 AT volume in male and female infants

\begin{tabular}{llll}
\hline $\begin{array}{l}\text { AT volume (mI); mean [95\% } \mathbf{~ C l}] \text {, after } \\
\text { adjustment for scan weight }\end{array}$ & Male (n=95) & Female (n=90) & p value \\
\hline Total AT & $746[718,774]$ & $841[812,869]$ & $<0.001$ \\
Abdominal superficial subcutaneous AT (SSAT) $102[97,107]$ & $122[117,128]$ & $<0.001$ \\
Non-abdominal SSAT & $540[519,560]$ & $606[586,627]$ & $<0.001$ \\
Abdominal deep subcutaneous AT (DSAT) & $14[13,15]$ & $18[17,20]$ & $<0.001$ \\
Non-abdominal DSAT & $12[11,13]$ & $13[12,14]$ & 0.008 \\
Abdominal internal AT (IAT) & $22[20,24]$ & $21[20,23]$ & 0.672 \\
Non-abdominal IAT & $56[53,60]$ & $59[55,63]$ & 0.310 \\
\hline
\end{tabular}

Conclusions Female newborn infants have higher total and subcutaneous AT, but similar internal abdominal AT compared to males. Longitudinal study is required to assess gender specific alterations in AT distribution during infancy and childhood, and may identify possible influences of internal abdominal AT development in males.

\section{OVERWEIGHT AND SCHOLASTIC ACHIEVEMENT IN CHILEAN SCHOOL-AGE CHILDREN}

doi:10.1136/archdischild-2012-302724.0361

${ }^{1} \mathrm{OC}$ Flores, ${ }^{\mathrm{V}} \mathrm{VF}$ Arias, ${ }^{1} \mathrm{CV}$ Ibaceta, 'YZ Orellana, ${ }^{2} \mathrm{E}$ Mena, ${ }^{2} \mathrm{VC}$ Arancibia, ${ }^{3} \mathrm{AF}$ Almagià, ${ }^{4} \mathrm{PA}$ Lizana, ${ }^{5} \mathrm{GI}$ Morales, ${ }^{1} \mathrm{RA}$ Burrows, 'DM Ivanovic. 'University of Chile, INTA; ${ }^{2}$ Pontifical Catholic University of Chile, Santiago; ${ }^{3}$ Pontifical Catholic University of Valparaíso; ${ }^{2}$ Pontifical Catholic University of Chile Catholic University of Valparaíso, Valparaíso; ${ }^{5}$ University of Chile, Faculty of Medicine, Santiago, Chile

Background and Aims Overweight has been linked to health problems and deteriorate school outcomes. Some studies show that overweight school-age children have lower scholastic achievement (SA) but the evidence is controversial. The aim of this study was to determine the association between overweight and SA in elementary fifth grade school children from the Chile's Metropolitan Region.

Methods This was an observational, cross-sectional and correlational study. Data were reported for 477 children of both sexes and from 26 schools (public, subsidized and non-subsidized). SA was measured with the Education Quality Measurement System test score (SIMCE), of national coverage. The current nutritional status was assessed through the body mass index z-score (z-BMI), controlling for dietary and physical activity behaviours, socio-economic status (SES, family and educational factors. Data were processed using descriptive statistics and multiple linear regressions from the Statistical Analysis System software (SAS).

Results Overweight children had not significantly lower SA compared with non-overweight children $(\mathrm{P}>0.05)$. SES $(\mathrm{P}<0.001)$, type of school $(\mathrm{P}<0.01)$, the number of repeated grades $(\mathrm{NRG})(\mathrm{P}<0.05)$ and parents' educational level $(\mathrm{P}<0.05)$ were the variables that correlated with SA. SES, the NRG and mother's educational level, were the variables with the greatest explanatory power for SA variance. The current nutritional status expressed as z-BMI did not contribute to explain SA variance.

Conclusion Current nutritional status was not associated with SA. SES, the number of repeated grades and mother's educational level could be strong predictors of SA.

Grant FONDECYT 1100431.

\section{HIGH INCIDENCE OF IRON DEFICIENCY IN HEALTHY YOUNG INFANTS IN THE NETHERLANDS: PRELIMINARY RESULTS OF THE IROSTAT STUDY}

doi:10.1136/archdischild-2012-302724.0362

'L Uijterschout, 'PP Teunisse, 'C Hudig, 'WW Rövekamp-Abels, ${ }^{2}$ SCAT Verbruggen, ${ }^{3,4} \mathrm{JB}$ van Goudoever, ' $\mathrm{F}$ Brus. 'Juliana Children's Hospital/HAGAZiekenhuis, The Hague; ${ }^{2}$ Erasmus MC - Sophia Children's Hospital, Rotterdam; ${ }^{3} \mathrm{VU}$ University Amsterdam, Amsterdam; ${ }^{4}$ Academic Medical Center, University of Amsterdam, The Hague, The Netherlands

Background Iron deficiency (ID) and iron deficiency anemia (IDA) during infancy are associated with poor neurological development. A food consumption survey in the Netherlands showed that the median iron intake of infants, aged 2 to 3 years, was below the advised adequate intake of $7 \mathrm{mg} /$ day.

Aim To investigate the iron status in healthy young infants in the Netherlands and to identify risk factors for ID.

Methods We conducted a multi centre, observational study in healthy young infants aged 0.5 to 3 years. We measured serum 